\title{
OP mit Herz-Lungen-Maschine: Höherer Ziel-Blutdruck hat keine Vorteile
}

Vedel AG et al. High-Target Versus Low-Target Blood Pressure Management During Cardiopulmonary Bypass to Prevent Cerebral Injury in Cardiac Surgery Patients. Circulation 2018; 137: $1770-1780$

Profitieren Patienten bei Eingriffen mit Herz-Lungen-Maschine, wenn ihr Blutdruck auf physiologischem (hohem) Niveau gehalten wird? Eher nicht, legt eine dänische Studie nahe.

Perioperative Schlaganfälle sind bei Patienten an der Herz-Lungen-Maschine häufig-Studien belegen 1-2\% klinisch manifeste und bis zu $50 \%$ stumme Hirninfarkte. Das Risiko steigt mit dem Alter der Patienten.

Oft fällt während solcher Eingriffe der Blutdruck auf sehr niedrige Werte. Wäre es besser, ihn medikamentös auf Normalniveau zu halten? Die „Washout“-Hypothese geht von der Vermutung aus, ein höherer arterieller Druck könnte Emboli besser aus der Hirnstrombahn spülen und so Infarkte vermeiden.

In der dänischen Studie wurden $197 \mathrm{~Pa}$ tienten randomisiert zwei Gruppen zugewiesen; in einer lag der mittlere arterielle Ziel-Blutdruck bei $70-80 \mathrm{~mm} \mathrm{Hg}$ (high target), in der anderen bei 40-50 $\mathrm{mm} \mathrm{Hg}$ (low target). Erreicht wurden die Werte durch Titrierung von Norepinephrin während des Eingriffs. Der Fluss durch den kardiopulmonalen Bypass be-

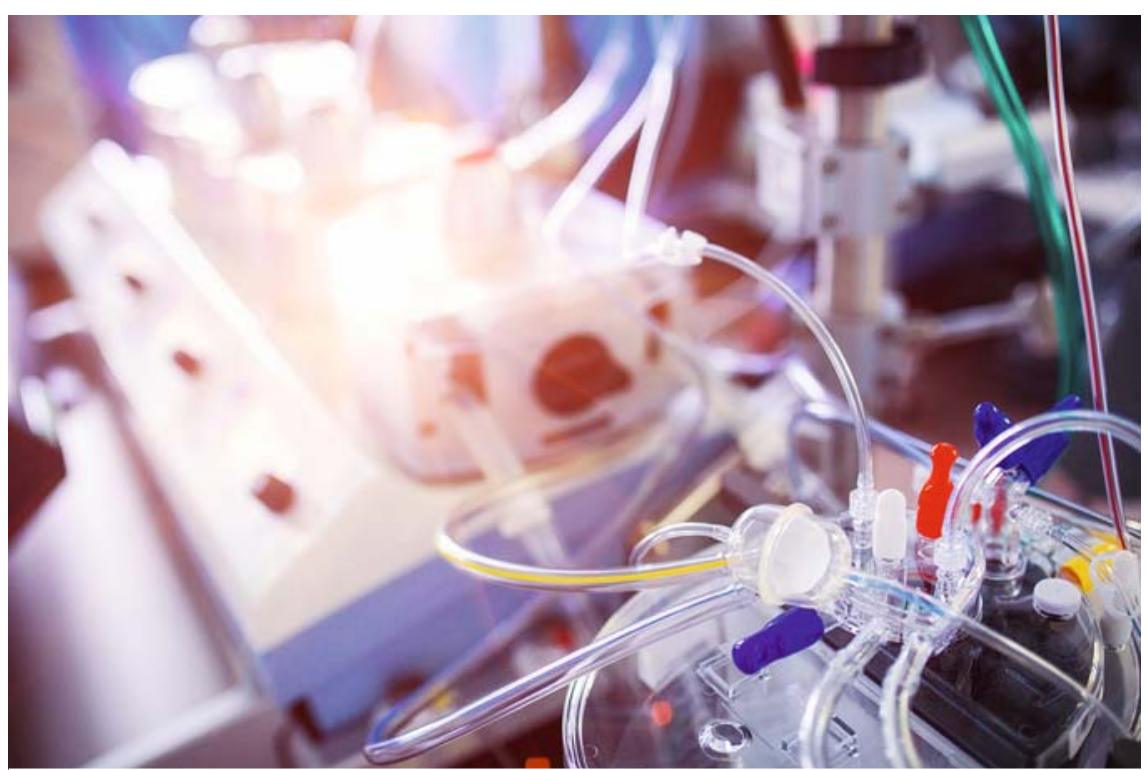

- Quelle: andrey_orlov/stock.adobe.com trug in beiden Gruppen konstant 2,4 I/m pro $\mathrm{m}^{2}$ Körperoberfläche.

\section{Perioperativ keine signifikanten \\ Unterschiede}

In der diffusionsgewichteten Magnetresonanztomografie wiesen 3-6 Tage nach dem Eingriff 52,8\% der Patienten in der Low-Target-Gruppe neue zerebrale Läsionen auf und 55,7\% der Patienten in der High-Target-Gruppe $(p=0,76)$. Das Volumen der Infarkte war mit 25 bzw. $29 \mathrm{~mm}^{3}$ in beiden Gruppen ähnlich $(p=0,99)$. Im Schnitt hatten die Patienten beider Gruppen 1 neue Läsion. Auch bei der Häufigkeit schwerer unerwünschter Wirkungen der Therapie zeigten sich keine signifikanten Unterschiede.

\section{Postoperativ mehr Schlag- anfälle bei High-Target}

Innerhalb des Bobachtungszeitraums von 30 Tagen waren klinisch manifeste Schlaganfälle zwar insgesamt selten, lagen in der High-Target-Gruppe aber höher als in der Low-Target-Gruppe (7\% vs.
$1 \%, p=0,059)$. Das Phänomen postoperativer Schlaganfälle nach großen HerzLungen-Operationen ist bekannt. Als Ursachen vermutet werden ein postoperatives Entzündungsgeschehen, eine gesteigerte Gerinnung oder neu aufgetretenes Vorhofflimmern. Ob ein großzügiger Gebrauch von Norepinephrin das Risiko für einen postoperativen Apoplex erhöht, wurde bislang nicht untersucht.

\section{FAZIT}

Ein höherer mittlerer arterieller ZielBlutdruck von $70-80 \mathrm{~mm} \mathrm{Hg}$ scheint bei Eingriffen mit extrakorporalem Kreislauf keinen messbaren Vorteil zu bringen. Weder die Zahl neuer zerebraler Infarkte noch ihr Volumen unterschieden sich im Vergleich mit Patienten, deren Ziel-Blutdruck während des Eingriffs bei $40-50 \mathrm{~mm} \mathrm{Hg}$ lag.

\section{Dr. Nina Drexelius, Hamburg}

\section{Kommentar}

Aus der vorgestellten Studie von Vedel et al. darf man bitte nicht vorschnell verallgemeinern, dass während einer extrakorporalen Zirkulation (EKZ) ein niedrigerer mittlerer arterieller Blutdruck (MAP) für das Gehirn nicht schlechter als ein höherer ist und damit auch akzeptiert werden kann. Das ist auch keinesfalls auf nichtherzchirurgische Eingriffe oder eine Schädigung anderer Organe zu übertragen. Angesprochen werden zwei aktuelle Themen: zerebrale Komplikationen und intraoperative Hypotonien.

In der Herzchirurgie und bei interventionellen Katheterinterventionen ist ein Schlaganfall eine gravierende, aber seltene (ca. 2\%) und multifaktorielle Komplikation. Er wird in erster Linie auf fokale und multifokale Embolisationen durch das Procedere selbst zurückgeführt [5]. Als weitere unabhängige Risikofaktoren für einen Schlaganfall sind u. a. ein höhe- 
res Alter, Hypertonie, Atherosklerose, Kombinationseingriffe, längere Bypasszeiten oder neu auftretendes Vorhofflimmern zu berücksichtigen [11]. Hypotonie und Hypoperfusion spielen in der Genese eines zerebralen Infarktes eine geringere Rolle [5]. Sehr viel häufiger sind die nicht fokalen oder unspezifischen Komplikationen wie Krampfanfälle, postoperatives Delir (POD) und postoperative kognitive Dysfunktionen (POCD) [5], die natürlich auch bei einem Schlaganfall auftreten können. Letztendlich ist die Pathophysiologie des perioperativen Schlaganfalls und einer zerebralen Schädigung nach herzchirurgischen Eingriffen nicht wirklich geklärt. Cheung [2] geht in seinem Editorial zur Studie von Vredel et al. auf Limitationen und wichtige Aspekte ein und gibt einen wunderbaren Einblick in die Komplexität dieser Thematik und die nicht einheitlichen Ergebnisse weiterer Studien. Eine noch aktuellere Arbeit zeigt sehr wohl einen Zusammenhang des Blutdrucks während EKZ mit dem Auftreten eines Schlaganfalles. Sun et al. fanden, dass während der extrakorporalen Zirkulation ein MAP von unter $65 \mathrm{~mm} \mathrm{Hg}$ für über 10 min mit einem zunehmenden Schlaganfallrisiko assoziiert ist [11] und das Risiko bei noch niedrigem MAP $<50 \mathrm{~mm} \mathrm{Hg}$ weiter steigt. Das reiht sich ein in die Tendenz der Literatur, dass eine EKZ mit einem höheren MAP gefahren werden sollte als mit dem traditionellen MAP von $50 \mathrm{~mm} \mathrm{Hg}$. Evidenz für einen bestimmten Zielwert während EKZ gibt es bislang nicht.

Auch für nicht- herzchirurgische Eingriffe gibt es bis heute keine Definition für eine Hypotension, die mit einer erhöhten Inzidenz von Organschäden und einem schlechteren Outcome assoziiert ist [4, 8,9,14]. Zunehmende Evidenz gibt es für die Korrelation des unteren MAP zu Organschäden und Outcome, wobei ein kausaler Zusammenhang noch nicht bewiesen ist $[4,14,9]$. Als Grenzwerte sind aktuell beschrieben: MAP<60-70 mm $\mathrm{Hg}$ [9], <55 mm Hg für > $10 \mathrm{~min}$ [12], $<80 \mathrm{~mm} \mathrm{Hg}>10 \mathrm{~min}$ und ein zunehmendes Risiko bei längerer Dauer mit MAP<60-65 mm Hg bzw. jede Hypotonie unter $50-55 \mathrm{~mm} \mathrm{Hg}$ [14].
Die unbefriedigende Datenlage wird darauf zurückgeführt, dass die vorliegenden Studien starke Unterschiede zeigen in der Patientenpopulation, den statistischen Modellen, den untersuchten Blutdruckgrenzen, der Art des chirurgischen Eingriffs oder in den Endpunkten [4].

Zudem variieren die Grenzen der Autoregulation patientenindividuell sehr stark und sind nicht zwingend für alle Organe gleich $(3,9)$. Von Mittelwerten eines MAP einer Population und aus Statistiken einer Studie darf man nicht auf den individuellen Patienten schließen $[4,13]$. Den einen Grenzwert des unteren MAP für alle Patienten gibt es eben nicht [1, 3]. Letztlich ist nicht nur die Schwere der Hypotonie allein, sondern auch ihre Dauer entscheidend $[3,9,10]$. Darüber hinaus nimmt nicht nur eine intraoperative Hypotonie [9] sondern- möglicherweise in viel stärkerem Maße- auch das postoperative Blutdruckmanagement $[4,8,9]$ Einfluss auf das Outcome, was bei bisherigen Studien gar nicht berücksichtigt wurde.

Für die Praxis - nicht nur während der EKZ - kann man schließen: jeder Patient hat seine eigenen Grenzen der Autoregulation und braucht einen individuellen MAP, nicht nur schwere und häufige, sondern auch längere Phasen unter dieser Druckgrenze müssen vermieden werden. Wie schön wäre es also, wenn wir die Autoregulation monitoren könnten!

Vielleicht kann die Verwendung von Scores unter Berücksichtigung von Schwere und Dauer der Hypotension bei der Erkennung kritischer Hypotonien helfen [10]. Ob uns ein zusätzliches zerebrales Monitoring wie die Bispectralanalyse (BIS) [7] oder die Nahinfrarotspektroskopie (NIRS) [7] weiter voran bringen können bleibt abzuwarten. Für die vielfach geforderten weiteren prospektiven Studien $[4,6,11,14]$ wäre wünschenswert, den Empfehlungen des „Neurologic Academic Research Consortium [5] zu folgen, die mit Definitionen, Klassifikationen und Beurteilungen neurologischer Endpunkte für Studien bei herzchirurgischen und kathetergestützten Interventionen eine standardisierte Grundlage vorgelegt haben.
Autorinnen/Autoren

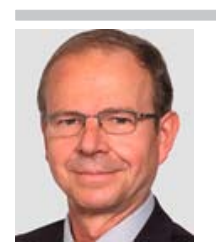

Prof. Dr. med. Uwe

Schirmer, Ruhr-

Universität Bochum

Literatur

[1] Brady K, Hogue CW. Intraoperative Hypotension and Patient Outcome. Does "One Fit All?" Anesthesiology 2013; 119: 495-497

[2] Cheung AT, Messé SR. Preventing Brain Injury After Cardiopulmonary Bypass Will Require More Than Just Dialing Up The Pressure. Circulation 2018; 137: 1781 - 1783

[3] Drummond JC. Blood Pressure and the Brain: How Low Can You Go? Anesth Analg 2019; 128; 759-771

[4] Ke JXC, George RB, Beattie WS. Making sense of the impact of intraoperative hypotension: from populations to the individual patient. $\mathrm{Br}$ ] Anaesth 2018; 121: 689-691

[5] Landsky AJ, Messé SR, Brickmann AM et al. Proposed Standardized Neurological Endpoints for Cardiovascular Clinical Trials. JACC 2017; 69: 679-691

[6] Langer T, Santini A, Zadek F et al. Intraoperative hypotension is not associated with postoperative cognitive dysfunction in elderly patients undergoing general anesthesia for surgery: results of a randomized controlled pilot trial. J Clin Anesth 2019: 52: 111-118

[7] Maheshwari A, McCormick PJ, Sessler DI et al. Prolonged concurrent hypotension and low bispectral index („double low“) are associated with mortality, serious complications, and prolonged hospitalization after cardiac surgery. Br J Anaesth 2017; 119: 40-49

[8] McEvoy MD, Gupta R, Koepke EJ et al. Perioperative Quality Initiative consensus statement on postoperative blood pressure, risk and outcome for elective surgery. $\mathrm{Br}$ ] Anaesth 2019; 122: 575-586

[9] Sessler DI, Bloonstone JA, Aronson S et al. Perioperative Quality Initiative consens statement on intraoperative blood pressure, risk and outcome for elective surgery. $\mathrm{Br}$ J Anaesth 2019; 122: $563-574$

[10] Stapelfeldt WH, Yuan H, Dryden JK et al. The SLUScore: A Novel Method for Detecting Hazardous Hypotension in Adult Patients Undergoing Noncardiac Surgical Procedures. Anesth Analg 2017; 124: 1135 - 1152

[11] Sun LY, Chung AM, Farkouh ME et al. Defining an Intraoperative Hypotension Threshold in Association with Stroke in Cardiac Surgery. Anesthesiology 2018; 129: 440-447 
[12] Tang Y, Zhu C, Liu J et al. Association of Intraoperative Hypotension with Acute Kidney Injury after Noncardiac Surgery in Patients Younger than 60 Years Old. Kidney Blood Press Res 2019; 29: 1 - 11

[13] Wesselink EM, Kappen TH, Torn HM et al. Intraoperative hypotension and the risk of postoperative adverse outcomes: a systematic review. $\mathrm{Br}$ J Anaesth 2018; 121: $706-721$ 